\title{
The self-deprivation phenomenon: Competition between appetitive rewards and electrical stimulation of the brain
}

\author{
RONALD R. ROSSI \\ Lyndon State College, Lyndonville, Vermont 05851 \\ and \\ ROBERT M. STUTZ \\ University of Cincinnati, Cincinnati, Ohio 45221
}

\begin{abstract}
Rats with electrodes implanted in several limbic midbrain structures were given a choice between electrical stimulation of the brain and food while food deprived, or electrical stimulation of the brain and water while water deprived. Rats that preferred brainshock over food also preferred brainshock to water. Noncontingent brain stimulation did not substantially reduce intake of either food or water. It is suggested that brainshock competes with biological reinforcers by virtue of its reinforcement potency rather than by its ability to activate a drive reduction mechanism.
\end{abstract}

The self-starvation phenomenon, which is a maladaptive preference, in a hungry animal, for positively reinforcing electrical stimulation of certain brain (ESB) areas rather than food, has been given several different theoretical interpretations. The earliest account of the phenomenon stemmed from the pioneering work of Olds (1958), who demonstrated that rats receiving rewarding hypothalamic ESB would self-stimulate to exhaustion, neglecting other bodily needs. According to Olds (1962), the highly rewarding properties of the ESB represented the activation of the neural systems that subserve conventional reward. Spies (1965) demonstrated that rats having rewarding electrodes in the so-called "feeding center" of the lateral hypothalamus (LH) preferred ESB to food in a discrete choice situation even during prolonged periods of food deprivation. However, rats having rewarding electrodes outside the LH showed no such preference. Spies (1965) suggested that in the case of the self-starving animals the ESB activated the neural mechanisms which signaled food intake, thereby "short circuiting" a hunger reduction mechanism. Routtenberg and Lindy (1965), whose report of self-starvation in a free responding situation appeared coincidentally with that of Spies, suggested that ESB in the lateral and posterior hypothalamic nuclear systems can cause temperature and glucose changes in addition to aug-

This research was supported by NSF Grants GB7629 and GB27654 and by a grant from the University of Cincinnati Research Council to Robert M. Stutz. Address reprints requests to Robert M. Stutz, Department of Psychology, University of Cincinnati, Cincinnati, Ohio 45221. mented motor activity, all of which can lead to a depression of food intake (Routtenberg \& Kuznesof, 1967).

This interpretation of self-starvation has been challenged. Stutz, Rossi, and Bowring (1971) found that the noncontingent administration of ESB to rats which had previously self-starved did not cause a decrease in food responding similar to that found in the same animals when the ESB was contingent upon a leverpress response. These results indicated that self-starvation was not the result of an artificial interference with the hunger regulatory mechanism. The alternative hypothesis proposed by Stutz et al. (1971) suggested that self-starvation reflected a simple preference for the immediately delivered and highly potent ESB reward over the food reward. Based on this interpretation, several predictions concerning the generality of the self-starvation phenomenon are possible. For instance, ESB which is preferred to food (thus producing self-starvation) should also be preferred to any other biologically relevant reinforcer. According to this view, the generality of self-starvation across drive states, or self-deprivation, should depend upon electrode locus and stimulation parameters only insofar as these factors determine the reinforcement strength of the stimulation. Thus, stimulation loci and parameters which produce self-starvation should produce other forms of self-deprivation in the same animal.

\section{METHOD}

The experiment consisted of two separate studies performed at different times. However, since the procedure of the original experiment and the procedure of the subsequent replication were 
nearly identical, they will be discussed as a single experiment with references made to the differences when appropriate.

\section{Subjects}

The animals were Sprague-Dawley albino rats 90 days of age at the time of electrode implantation.

\section{Surgery}

The rats were anesthetized with sodium pentobarbital $(45 \mathrm{mg} / \mathrm{kg})$ supplemented with $0.1 \mathrm{cc}$ atropine sulfate in order to alleviate respiratory congestion. In the original experiment, a single bipolar stimulating electrode, $0.020 \mathrm{in}$. across both poles (Plastics Products Company, Roanoke, Virginia, No. 303-018-312) was stereotaxically implanted in each animal. The coordinates given for the implantation of the electrodes were "flat-head" coordinates; that is, the surface of the animal's skull was in a horizontal position in the stereotaxic instrument. In seven animals, the electrodes were aimed at the medial forebrain bundle (MFB) as it passes through the lateral hypothalamic area (coordinates: $5 \mathrm{~mm}$ posterior to bregma, $1.5 \mathrm{~mm}$ lateral to the midline suture, $8.5 \mathrm{~mm}$ below the dorsal surface of the skull); in five animals, the electrodes were aimed at the diagonal band of Broca (DBB) in the septal region (coordinates: $0.5 \mathrm{~mm}$ anterior to bregma, $0.5 \mathrm{~mm}$ lateral, and $7.0 \mathrm{~mm}$ ventral); and in two animals, the electrodes were aimed at the MFB as it passes more diffusely through the lateral preoptic area (coordinates: $0.0 \mathrm{~mm}$ from bregma, $2.5 \mathrm{~mm}$ lateral, $8.0 \mathrm{~mm}$ ventral). In the replication experiment, a monopolar electrode was used. It consisted of a length of 26-ga Nichrome wire, insulated except at the tip, and crimped to an Amphenol micro-miniature connector. The reference electrode consisted of a 3-mm length of uninsulated wire crimped to the skull connector. In all eight animals in the replication, the monopolar electrodes were aimed at the MFB as it passes through the lateral hypothalamic area (coordinates: $4.5 \mathrm{~mm}$ posterior, $1.5 \mathrm{~mm}$ lateral, $8.5 \mathrm{~mm}$ ventral) and the reference electrode was placed $3-4 \mathrm{~mm}$ posterior to the stimulating electrode. The electrodes were attached to the skull with optical screws and dental acrylic, and 1 week was allowed for recovery from surgery.

Self-stimulation tests. All animals were tested for selfstimulation in a wood and Plexiglas box which measured $30 \times 20 \times$ $25 \mathrm{~cm}$. Depression of a lever (Scientific Prototype Manufacturing Corporation, New York, PLS-100), which was mounted on one wall, resulted in a single $300-\mathrm{msec}$ train of $100-\mathrm{Hz}$ sine-wave current generated by a Grass stimulator (Model S-4). Duration of the brain stimulation was regulated by a Hunter Interval Timer which also pulsed an electrical counter. The animals were tested for self-stimulation for $10 \mathrm{~min}$ each day for 5 days. During these daily 10 -min sessions, the current level was adjusted to produce maximum leverpressing rates in each animal without disruptive motor involvement or convulsions. Animals that did not leverpress for ESB more than 50 times in the 10-min sessions were dropped from the experiment. The remaining animals were run for 30-min sessions on 5 additional days in order to establish stable base rates of responding. Of the 14 animals implanted in the original experiment, 8 reached the criterion of at least 50 responses in $10 \mathrm{~min}$. Three of these animals had hypothalamic electrodes, $3 \mathrm{had}$ septal electrodes, and 2 had preoptic electrodes. In the replication, all of the 8 animals with LH electrodes showed self-stimulation.

\section{ESB/Water Competition}

Free drinking phase. After stable self-stimulation rates had been established, the animals were placed on a $231 / 2$-h water-deprivation schedule. They were trained to leverpress in daily 30 -min sessions to obtain water on a continuous reinforcement schedule. This training was conducted in the same box used for self-stimulation testing, except that the water lever was mounted on the wall directly across from the lever that delivered ESB. Depression of this second lever activated a liquid dispenser (Scientific Prototype Manufacturing Company, New York, Model L11) which delivered
$0.10 \mathrm{ml}$ of water to a cup mounted on the wall next to the lever. The ESB lever was inactive during this phase of training. Water was available to the animals only during these daily sessions, and training was continued until each animal maintained stable leverpressing rates for at least 3 consecutive days. When this point was reached, competition testing began.

Competition phase. During this phase, which lasted for 4 days, both the ESB and the water levers were activated and the animals were allowed to respond on either lever for the appropriate reward. The exact temporal pattern of each day's responding for ESB was recorded for each animal on a 30-min continuous magnetic tape for playback during a later phase of the experiment.

Recovery phase. During this phase, only the water lever was active and free responding for water was allowed. Recovery was considered complete when stable rates of responding for water were again observed.

Noncontingent stimulation phase. Following the recovery period, the noncontingent phase was begun. During this part of the experiment, which lasted for 4 days, only the water lever was active. ESB was presented noncontingently by playing back the magnetic tapes on which were recorded each animal's daily pattern of ESB responding during competition.

\section{ESB/Food Competition}

After a 2-week recovery period with food and water continuously available, the same animals were subjected to a second competition test. The procedure involved in the food/ESB competition was identical to that previously described for water, except that the animals were food deprived and depression of the lever resulted in the delivery of a 45-mg Noyes food pellet. All food was obtained in this manner during daily 30 -min sessions, and all animals were required to maintain a stable weight level for 3 consecutive days before competition testing was initiated. The change in the reinforcement from water to food was the only procedural change that occurred from the water/ESB competition test.

Tests for stimulus-bound behavior. One week following completion of the food/ESB competition tests, the animals were subjected to tests for stimulus-bound behavior. The animals were individually placed into the test chamber, which contained a dish of food pellets, a water bottle, and some small wooden blocks, and allowed to explore the chamber for a 5 -min period. At the end of this familiarization period, brain stimulation was turned on for $30 \mathrm{sec}$ at the same intensity used during the previous tests. The 30-sec train of stimulation was followed by $60 \mathrm{sec}$ of "no stimulation." In all, 10 stimulation periods were presented. Records were made of any eating, drinking, or gnawing responses elicited by the stimulation. Although self-stimulation and stimulusbound behavior are usually controlled by different stimulation parameters, this test was included to insure that the parameters actually used did not elicit consumatory behaviors.

\section{Histology}

When the tests for stimulus-bound behavior were completed, the animals were sacrified with an overdose of Nembutal and perfused with $.9 \%$ saline followed by Formalin. The brains were removed, embedded in celloidin, sliced at a thickness of $50 \mu$, and stained with cresylfast violet. The electrode placements were determined using the de Groot atlas (de Groot, 1959).

\section{RESULTS}

Since Routtenberg and Lindy (1965) and Stutz et al. (1971) used only food reinforcement, their criteria for determining self-deprivation were based primarily on weight measures. However, water, as well as food, was used as a reinforcement in the 
present experiments. To determine the criteria for self-deprivation in the present series of experiments, the rate of leverpressing for the primary reward (i.e., food or water) during competition was expressed as a percentage of the rate of leverpressing during the precompetition phase. An animal was considered a self-depriver if its leverpressing rate for a primary reward on all days of competition was less than $50 \%$ of the mean precompetition rate and if no increase in responding for the primary reward occurred across the 4 days of competition. If the competition rate was more than $50 \%$ of the precompetition rate or if a substantial increase occurred across the 4 days, the animal was considered to be a nondepriver.

According to these criteria, eight of the animals were classed as self-deprivers during the water/ESB competition. The remaining eight animals maintained stable rates of leverpressing for water during this portion of the experiment and were classed as nondeprivers. The same eight animals that deprived themselves of water also deprived themselves of food during the food/ESB competition, and the eight water-maintaining animals also maintained stable leverpressing rates for food. In no case did an animal that self-deprived for water fail to self-deprive for food; and, similarly, in no case did an animal that maintained stable rates for water fail to maintain stable rates for food.

Mean leverpress rates for water for the selfdeprivers and the nondeprivers during the pretraining, competition, recovery, and noncontingent phases of the experiment are shown in Figure 1. Mean leverpress rates for food during these same phases are shown in Figure 2. It is apparent from these figures

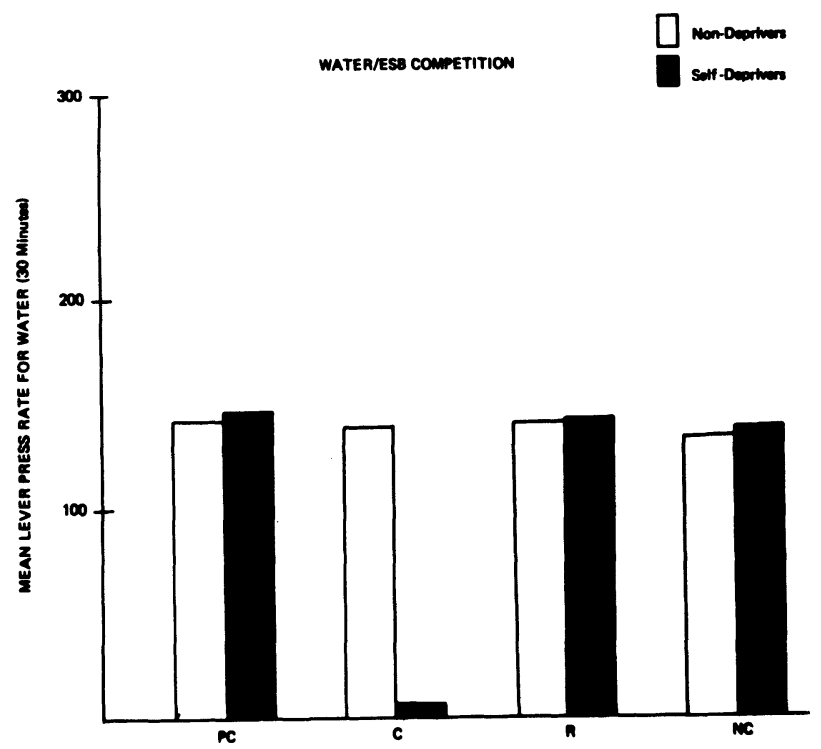

Figure 1. Mean leverpress rates for water for the self-deprivers and the nondeprivers during the precompetition (PC), competition (C), recovery (R), and noncontingent (NC) phases of the experiment.

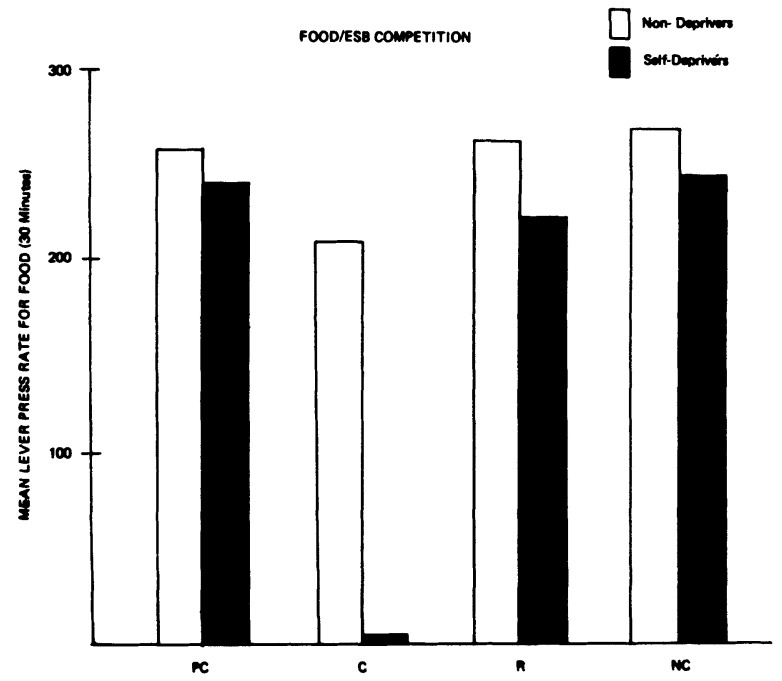

Figure 2. Mean leverpress rates for food for the self-deprivers and the nondeprivers during the precompetition (PC), competition (C), recovery (R), and the noncontingent (NC) phases of the experiment.

that the self-depriving animals almost totally neglected food and water during the competition phases. Comparisons of the mean response rate for food or water during the recovery period with the mean response rate during the noncontingent period revealed that noncontingently delivered ESB did not significantly reduce leverpressing for either primary reward in either the self-depriving (SD) or the nondepriving (ND) groups; [ND-food: $t(7)=0.54$; ND-water: $t(7)$ $=1.97$; SD-food: $\mathrm{t}(7)=2.88, \mathrm{p}<.05^{1}$; SD-water: $t(7)=1.89$ ]

Not a single animal showed evidence of any of the three responses measured during the tests for stimulusbound behavior. Neither eating, drinking, nor gnawing could be elicited by the administration of long durations $(30 \mathrm{sec})$ of stimulation adjusted to the current level used in the self-stimulation tests.

A comparison of the precompetition self-stimulation rates revealed that the mean rate of leverpressing for ESB for the self-deprivers was significantly higher than that of the nondeprivers $[t(7)=7.02, p<.005]$.

The results of the histological analysis are presented in Figure 3. The nondepriving animals, which are represented in the figure by circles, had electrodes located in a variety of limbic system structures, including the diagonal band of Broca $(n=3)$, the preoptic area $(n=2)$, and around the lateral hypothalamic area $(n=3)$. The self-deprivers had electrodes falling directly in the MFB $(n=7)$ or in the ventral tegmental area of Tsai $(n=1)$. None of the nondeprivers had an electrode in the MFB.

\section{DISCUSSION}

The self-deprivation phenomenon has been demonstrated when ESB is placed in competition 


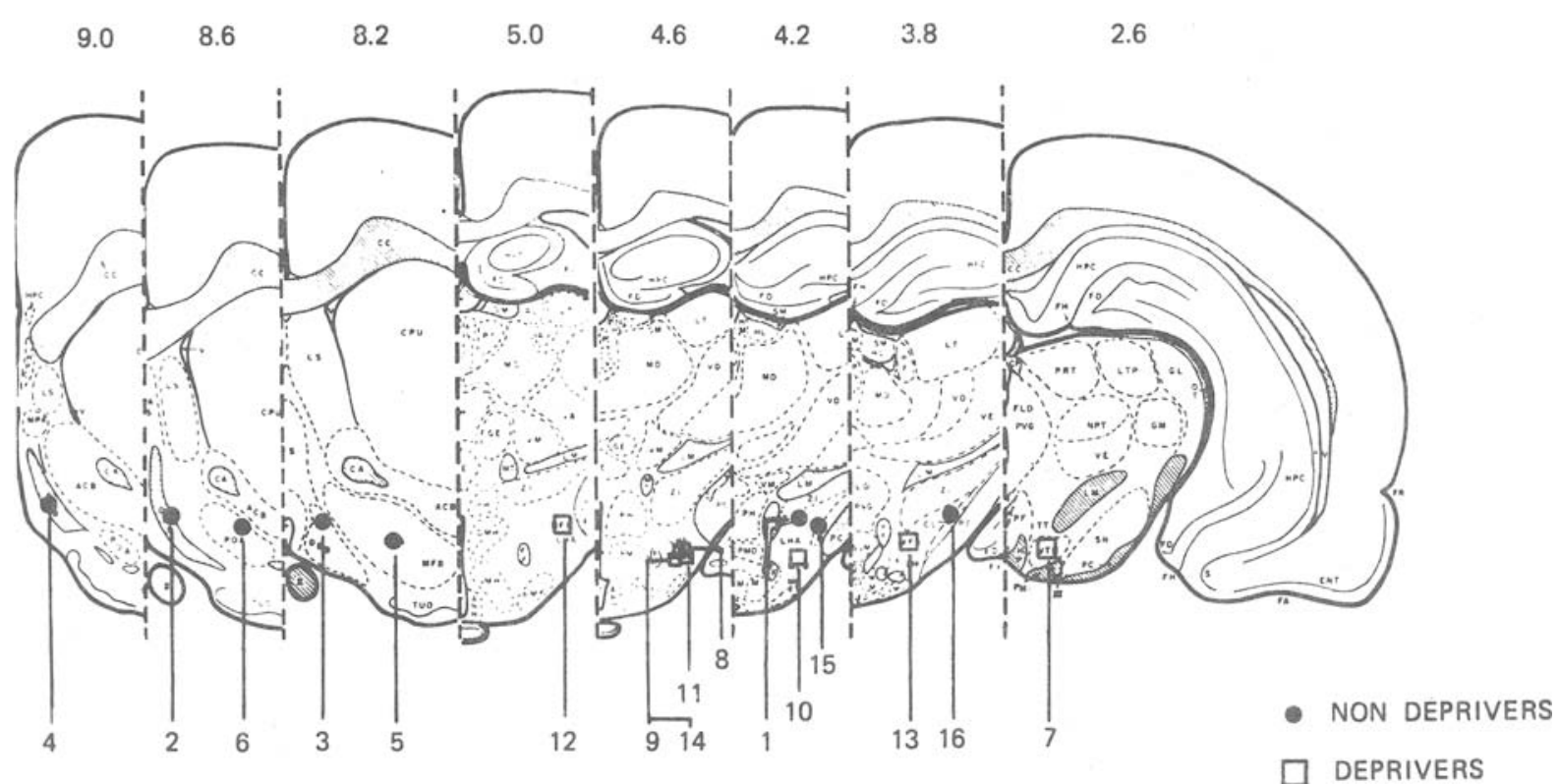

Figure 3. Electrode placements for each subject. (Bipolar electrodes: No. 1-8; monopolar electrodes: No. 9-16.) The numbers at the top of the figure correspond to the plates in the de Groot atlas.

with food reward in food-deprived animals (Eckert \& Lewis, 1967; Olds, 1958; Routtenberg \& Bulloch. 1971; Routtenberg \& Kuznesof, 1967; Spies, 1965; Stutz et al., 1971), water reward in water-deprived animals (Falk, 1961; Morgan \& Mogenson, 1966; Valenstein \& Beer, 1962), and salt reward in adrenalectomized, salt-deprived animals (Eckert \& Lewis, 1967). The results of the present study, which are consistent with these previous demonstrations of selfdeprivation, indicate that the self-deprivation phenomenon is independent of the specific drive state and primary reward placed in competition with ESB; animals that deprive themselves of food also deprive themselves of water. The outcome of the noncontingent phases of the experiment are consistent with those obtained by Stutz et al. (1971); ESB which is freely administered to an animal that had previously self-deprived does not cause the same reduction in leverpressing for the primary reward that occurs when the ESB is made contingent upon the leverpressing response during competition with the primary reward.

Although the interpretion of self-deprivation based solely on the relative reward value of ESB as put forth by Stutz et al. (1971) readily accounts for the nonspecificity of the phenomenon, alternative explanations are available and should be considered.

It has been demonstrated, for example, that several different classes of responses can be elicited by stimulation through a single electrode (e.g., Roberts, 1969; Valenstein, Cox, \& Kakolewski, 1968). Although the reason for this plasticity of elicited responses is still open to question, it does indicate that stimulation through a single electrode in the hypothalamic region may affect components of more than one drive system. Thus, it could be argued that the nonspecificity of self-deprivation is due to the simultaneous interference with both food and water regulatory mechanisms by the stimulation. However, the results of the present study are not consistent with such an interpretation. In the first place, the tests for stimulus-bound behavior were negative. Thus, there is no evidence that the electrodes were stimulating either the hunger or the thirst drive systems. Second, the lack of effect of the noncontingently administered ESB on either food or water intake indicates that self-deprivation is not due to any drive-reducing, transient satiety, or consumatory response suppression functions of the ESB.

As in previous self-starvation studies (Routtenberg \& Bulloch, 1971; Routtenberg \& Lindy, 1965; Stutz et al., 1971), the brain sites which supported the phenomenon were primarily limited to the MFB, which is considered to be a major neural focus of the self-stimulation or positive reinforcement system (Stein, 1968). Thus, it follows that the intensity and purity of the reward produced by the stimulation should be a function of the number of MFB fibers stimulated at any one time. This number would be greatest if the electrode was aimed at the MFB as it passes through the $\mathrm{LH}$ as a tightly compacted bundle. In the septal and preoptic areas where the MFB has separated into several smaller bundles of fibers, the intensity and purity of the reward would not be as great. The ventral tegmental placements may produce intensely rewarding effects because it is in this region 
that the ipsilateral trajectories of the MFB converge (Nauta, 1960). The results of the competition tests indicate that stimulation of the MFB in the lateral hypothalamic and ventral tegmental areas has a greater reward value than primary rewards, thus producing a general self-deprivation phenomenon. Unfortunately, reward strength and electrode placement were confounded in the present experiments. In order to rule out the importance of locus per se, it would be necessary to locate one or more brain sites which support self-stimulation rates equal to those obtained from the MFB or VTN. Successful attempts to accomplish this have not been reported.

\section{REFERENCES}

DE GROOT, J. The rat forebrain in stereotaxic coordinates. Der Koninklijke Nederlandse Akademie van Wetenschappen, afd. Natuurkunde, 1959, 52, 1-40.

ECKERT, B., \& LEwIs, M. Competition between drives for intracranial stimulation and sodium chloride by adrenalectomized $\mathrm{NaCl}$-deprived rats. Journal of Comparative and Physiological Psychology, 1967, 64, 349-352.

FALK, J. L. Septal stimulation as a reinforcer of and an alternative to consummatory behavior. Journal of the Experimental Analysis of Behavior, 1961, 4, 213-217.

Morgan, C. W., \& Mogenson, G. J. Preference of water deprived rats for stimulation of the lateral hypothalamus rather than water. Psychonomic Science, 1966, 6, 337-338.

Nauta, W. J. H. Some neural pathways related to the limbic system. In E. R. Rainey \& D. S. O'Doherty (Eds.), Electrical studies on the unanesthetized brain. New York: Hoeber, 1960. Pp. 1-16.

OLds, J. Satiation effects in self-stimulation of the brain. Journal of Comparative and Physiological Psychology, 1958, 51, 675-678.

OLds, J. Hypothalamic substrates of reward. Physiological Review, 1962, 42, 554-604.

RoBERTs, W. W. Are hypothalamic motivational mechanisms functionally and anatomically specific? Brain, Behavior and Evolution, 1969, 2, 317-342.

Routtenberg, A., \& Bulloch, G. C. Self-starvation and rewarding brain stimulation: Effects of chlorpromazine and pentobarbital. Learning and Motivation, 1971, 2, 83-94.

Routtenberg, A., \& Kuznesof, A. W. Self-starvation of rats living in activity wheels on a restricted feeding schedule. Journal of Comparative and Physiological Psychology, 1967, 64, 414-421.

RouttenberG, A., \& Lindy, J. Effects of rewarding septal and hypothalamic stimulation on bar pressing for food under conditions of deprivation. Journal of Comparative and Physiological Psychology, 1965, 60, 158-161.

SPIES, G. Food versus intracranial self-stimulation reinforcement in food deprived rats. Journal of Comparative and Physiological Psychology, 1965, 60, 153-157.

STEIN, L. Chemistry of reward and punishment. In D. H. Efron (Ed.), Psychopharmacology: $A$ review of progress, 1957-1967. Washington, D.C: U.S. Government Printing Office, 1968. Pp. 105-123.

Stutz, R. M., Rossi, R. R., \& Bowring, A. M. Competition between food and rewarding brain shock. Physiology \& Behavior, 1971, 7, 753-757.

VAlenstein, E. S., \& BeER, B. Reinforcing brain stimulation in competition with water reward and shock avoidance. Science, 1962, 137, 1052-1054.

Valenstein, E. S., Cox, V. C., \& Kakolewski, J. W. Modification of motivated behavior elicited by electrical stimulation of the hypothalamus. Science, 1968, 159, 1119-1121.

\section{NOTE}

1. The significant change in the self-deprivers' leverpress rate for food in the noncontingent phase represents a singificant increase, not a decrease. This increase was not, however, significantly above precompetition levels $[\mathrm{t}(7)=0.389]$, and it suggests that the self-depriving animals may need a longer recovery period than the nondeprivers because of their greater weight loss.

(Received for publication November 10, 1977; revision accepted January 20, 1978.) 